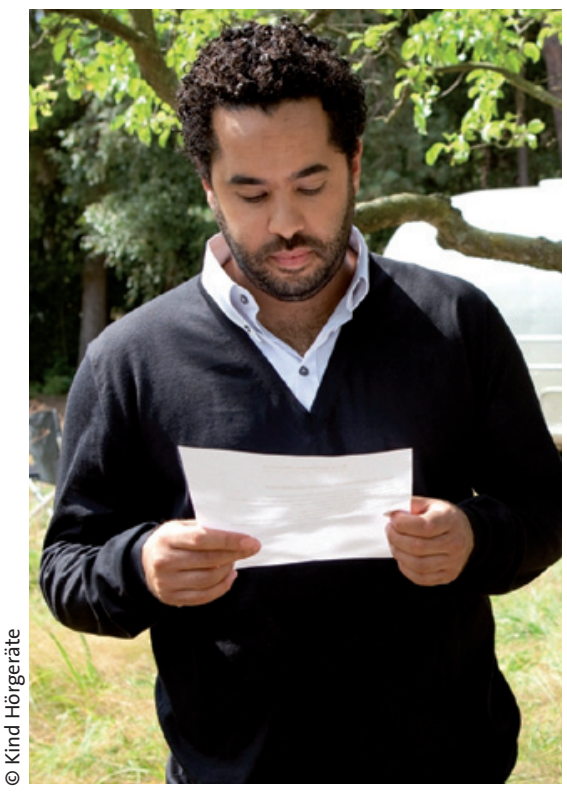

Adel Tawil beim Dreh der TV-Spots für die Kampagne

\title{
Mehr Aufmerksamkeit für Hörprobleme
}

- Rund 15 Millionen Menschen in Deutschland hören schlecht, aber nur 3 Millionen Menschen tragen Hörgeräte. Viele Betroffene verdrängen nämlich ihren Hörverlust, so dass Schwerhörigkeit oft sieben bis zehn Jahre zu spät erkannt und behandelt wird. Ein Problem dabei: Das jahrelange schlechte Hören bleibt nicht ohne Folgen. Die nicht mehr wahrgenommenen Töne „verlernt" das Gehirn, die Anpassung von Hörgeräten gestaltet sich dann sehr viel aufwändiger und langwieriger, da bestimmte Geräusche und Frequenzen erst wieder neu erlernt werden müssen. Hinzu kommt, dass unbehandelte Schwerhörigkeit die Teilnahme am gesellschaftlichen Leben erschwert, so dass die Betroffenen oft sogar in die soziale Isolation geraten.
Um diesen Problemen entgegenzuwirken und für das Thema Schwerhörigkeit zu sensibilisieren, hat die Hörgeräteakustik-Firma Kind die Kampagne „Das ganze Leben hören" gestartet. Kunden der Firma, darunter so prominente Menschen wie Schauspieler Michael Degen, der ein Hörgerät trägt, und der Sänger der Band Ich+lch, Adel Tawil, der Hörschutz benutzt, machen sich dabei für das Thema Hören stark.

Die Bekenner-Kampagne von Kind soll Menschen Mut machen, zum Hörtest zu gehen, Hörgeräte zu testen und allen anderen zu verdeutlichen, wie wichtig es ist, das Gehör zu schützen.

gz

\section{Pflege und Schutz für Stimme und Rachen- schleimhaut}

— Reizhusten und Räusperzwang aber auch zum Beispiel eine Refluxerkrankung können der Rachenschleimhaut und der Stimme gewaltig zusetzen. Soweit möglich sollten stets die Ursachen für diese oft sehr hartnäckigen Beschwerden ausgeschaltet werden, sei es durch gesunde Lebensführung oder die Therapie der Grunderkrankung. Darüber hinaus kann der Schutz der empfindlichen Schleimhäute in Kehlkopf und Rachen wertvolle Hilfe leisten und für Linderung sorgen. Hierzu steht seit Oktober Natürliches Emser Salz in Form eines Sprays zur Verfügung (Emser ${ }^{\circledR} \mathrm{Hals}$ - und Rachenspray). Eine Kombination aus Mineralstoffen und Spurenelementen löst auf natürlicher Basis festsitzende Beläge und mindert eine Übersäuerung der Schleimhäute, die ebenfalls zu Stimmstörungen führen kann. Das Spray ist frei von Konservierungsmittel und ätherischen Ölen und auch für Kinder, während der Stillzeit und für Schwangere geeignet.

gz

Nach Informationen von Siemens \& Co

\section{Versorgung mit Mikronährstoffen bei stressbedingtem Tinnitus}

— Jeder dritte Deutsche leidet unter Dauerstress und ganze neun Prozent der Dauergestressten litten zudem unter Tinnitus, berichtete Dr. Stephanie Grabhorn, Frankfurt auf einer Fachveranstaltung des Unternehmens Orthomol in Hamburg. Wie chronischer Stress zur Tinnitusentstehung und -verstärkung beitrage, erläuterte PD Dr. Birgit Mazurek: Die vermehrte Ausschüttung von Kortisol führe über eine erhöhte Glukoneogenese, Lipolyse und Proteolyse zur vermehrten Energiebereitstellung. Darüberhinaus werde eine exzessive Glutamat- und damit letzlich eine erhöhte Calciumausschüttung getriggert. Da sich in der Cochlea viele Glukokortikoidrezeptoren finden, kann es zu einer verstärkten Bildung von Aktionspotenzialen kommen, die im Gehirn als Hyperaktivität wahrgenommen und als Tinnitus interpretiert werden können. Als Glutamatantagonist sei insbesondere die Therapie mit dem Mikronährsotff Magnesium zu empfehlen, so Mazurek. Speziell zur diätetischen Unterstützung von stressbezogenen Innenohrerkrankungen, insbesondere Tinnitus und Hörsturz steht Orthomol Audio ${ }^{\circledR}$ zur Verfügung, eine ergänzend

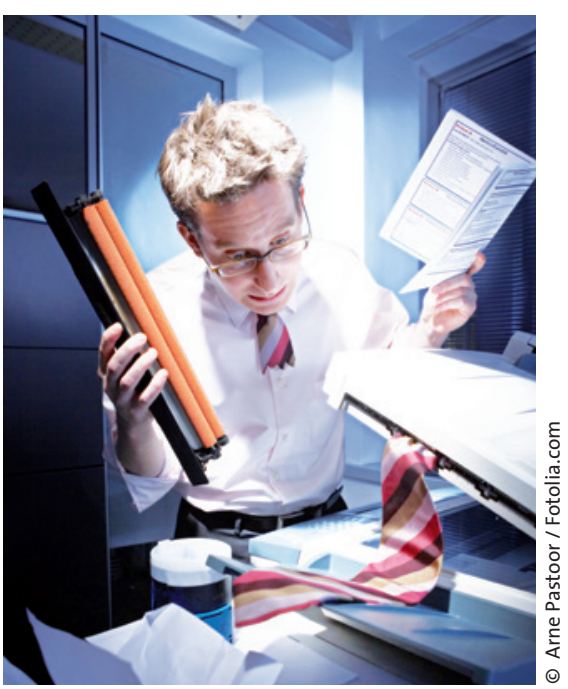

Chronischer Stress kann zur Entstehung von Tinnitus beitragen.

bilanzierte Diät mit Mikronährstoffen, die oxidativem Stress entgegenwirken, die Gefäßfunktionen und den Energiestoffwechsel unterstützen.

gz

Nach Informationen von Orthomol 